\title{
Instantaneous Frequency Estimation of Multi-Component Non- Stationary Signals using Tunable-Q Wavelet Transform and Time-Varying Auto Regressive Model
}

\author{
G. Ravi Shankar Reddy ${ }^{1}$ and Rameshwar $\mathrm{Rao}^{2}$ \\ ${ }^{1}$ Dept.of ECE, CVRCollege of Engineering, Hyderabad, India \\ ${ }^{2}$ Ex-Vice Chancellor, JNT University, Hyderabad, India \\ ${ }^{1}$ ravigosula_ece39@yahoo.co.in, ${ }^{2}$ rameshwar_rao@hotmail.com
}

\begin{abstract}
In this paper, we present a new parametric technique for Instantaneous Frequency (IF) estimation of multi-component non-stationary signals using joint application of Tunable-Q Wavelet Transform (TQWT) and Time-Varying Auto Regressive(TVAR) model. The proposed technique is composed of two steps:1) Multi-component signal is decomposed into number of mono-component signals using TQWT. 2) IF of each extracted mono-component signal is estimated using TVAR model. In the first step: J level TQWT decomposes the multi-component signal into $(J+1)$ number of wavelet coefficients sub bands. It is observed that the distribution of signal energy with respect to $T Q W T$ sub bands has clustering (or) grouping property. Where each group corresponding to a mono-component signal. Applying Inverse TQWT to each sub band results to $(J+1)$ reconstructed time domain signals. Adding the reconstructed time domain signals of each cluster a mono-component signal is extracted. In the second step: TVAR model is applied to each extracted mono-component signals to compute their instantaneous frequencies. In TVAR modeling approach the time varying parameters are expanded as a linear combination of basis functions. In this paper, the TVAR parameters are expanded by a discrete cosine basis functions. IF is extracted from the time-varying autoregressive parameters by calculating the angles of the estimation error filter polynomial roots. Simulation results are included to show the effectiveness of the proposed method.
\end{abstract}

Keywords: Tunable- $Q$ wavelet transform; Basis function, Instantaneous Frequency, Multi component non stationary signal, Time-varying Auto Regressive model

\section{Introduction}

Many signals encountered in practice, such as speech signal, ECG signal, and EEG signal etc., are multi-component non-stationary signals. The extraction of monocomponent signals from a multi-component signal is referred to as decomposition of the multi-component signal. The decomposition of a multi-component signal in to monocomponent signals finds application in signal analysis, modeling and classification [1].

A method based on the Gabor filtering of a multi-component signal was proposed in [1] but introduces distortion in the extracted mono-component signals. This drawback of [1] was rectified in [2] by employing Fourier-Bessel based component separation. However, to extract mono-component signals, the method requires manual determination of the range of FB coefficients.

An adaptive method, Empirical mode decomposition (EMD) has been proposed in [3] to decompose a multi-component non-stationary signal into a set of intrinsic mode functions (IMFs) using the sifting process, but the IMFs are not the mono-component signals. 
Recently, a wavelet transform, named tunable Q-factor wavelet transform (TQWT), is proposed for discrete-time signal analysis for which the Q-factor is easily tunable [4-5]. Joint application of TQWT and Morphological component analysis (MCA) is proposed to decompose the signals into a high-resonance component and low-resonance component [6-7].It can be concluded from the literature review that the capability of TQWT to decompose multi-component non stationary signal into mono-component non-stationary signals has yet to be explored. In this paper a new analysis is proposed based on TQWT decomposition and reconstruction. Using TQWT decomposition and reconstruction the multi-component non stationary signal is decomposed into a number of mono-component signals

In signal processing, the estimation of IF is a kind of very important for non-stationary multi-component signals i.e, signals whose spectra change with time. Firstly, this paper adopts TQWT decomposition to decompose multi-component non-stationary signal in to mono-component signal. Then using TVAR model is used to obtain IF of monocomponent.

TVAR Model is applied to each mono- component signal to estimate IF of nonstationary signal. The time-varying frequencies of the non-stationary signals are contained in the time-varying coefficients of the TVAR model. Extracting the frequency information consists of two steps: first, the TVAR parameter estimation at time n, and then frequency calculation. Approaches for estimating the TVAR parameters may be classified into two categories; namely adaptive algorithms and the basis function method [8].

It was demonstrated that adaptive algorithms are failed to track the time-varying frequency of the signal, if the frequency changed very fast [8].The basis function method in which time-varying parameters are expressed as a linear combination of constants multiplied by basis functions. The time varying frequencies are extracted from the time varying auto regressive parameters by calculating the angles of the estimation error filter polynomial roots. Selection of basis and Model order determination are the two issues in TVAR Model, detail discussions can be found elsewhere [9].

The paper is organized as follows: a brief overview of the Tunable-Q Wavelet Transform is presented in Section 2, TVAR Model is briefly reviewed in section 3, and Simulation results are shown in section 4. Finally, conclusion is presented in Section 5.

\section{Tunable-Q Wavelet Transform}

\subsection{Single-Stage TQWT}

Wavelet transform with a relatively high Q-factor is suitable for processing oscillatory signals, such as speech signals and mechanical signals. However, when processing signals with no oscillatory behavior, it requires wavelet transform with a relatively low Q -factor. In other words, when using Wavelet Transform, we should select a proper wavelet base according to the oscillatory behavior of the signal to which the wavelet transform is applied. Selesnik recently developed a TQWT [4]. One of the most important advantages of the TQWT is that it is more flexible because the Q-factor can be easily tuned for the concerned signal.

The TQWT is basically a fully-discrete wavelet transform. The concept of the TQWT is simple. TQWT is implemented using an reversible over sampled filter bank with real valued scaling factors. The filters, employed in TQWT have rational transfer functions that are more computationally efficient and are easily specified directly in the frequency domain [4-5]. The single stage Analysis and Synthesis filter bank for TQWT is shown in Figure 1 . 
For perfect reconstruction, the frequency responses $H_{0}(\omega)$ and $G_{0}(\omega)$ must be chosen so that the reconstruction signal $y(n)$ equals to input signal $x(n)$. The low-pass filter $H_{0}(\omega)$ and the high-pass filter $G_{0}(\omega)$ are constructed as follows [4]:

$$
\begin{aligned}
& H_{0}(\omega)=\left\{\begin{array}{l}
1, \text { for }|\omega| \leq(1-\beta) \pi \\
\theta\left(\frac{\omega+(\beta-1) \pi}{\alpha+\beta-1}\right), \text { for }(1-\beta) \pi \leq|\omega| \leq \alpha \pi, \\
0, \text { for } \alpha \pi \leq|\omega| \leq \pi
\end{array}\right. \\
& G_{0}(\omega)=\left\{\begin{array}{l}
0, \text { for }|\omega| \leq(1-\beta) \pi \\
\theta\left(\frac{\alpha \pi-\omega}{\alpha+\beta-1}\right), \text { for }(1-\beta) \pi \leq|\omega| \leq \alpha \pi, \\
1, \text { for } \alpha \pi \leq|\omega| \leq \pi
\end{array}\right.
\end{aligned}
$$

Where $\theta(\omega)$ is the Daubechies Filter frequency response with two vanishing moments, is used to construct the transition bands of $H_{0}(\omega)$ and $G_{0}(\omega)$ which can be expressed as follows [4]:

$$
\theta(\omega)=0.5(1+\cos \omega) \sqrt{2-\cos \omega} \text { for }|\omega| \leq \pi
$$

Equations (1) and (2) indicate that the low-pass filter and the high-pass filter can be determined if a pair of scaling parameters $(\alpha, \beta)$ is given. It can be checked that[4]the low-pass filter $H_{0}(\omega)$ and the high-pass filter $G_{0}(\omega)$ meet the perfect reconstruction requirement $\left|H_{0}(\omega)\right|^{2}+\left|G_{0}(\omega)\right|^{2}=1, L P S \alpha$ and $H P S \beta$ in Figure 1, represent low pass scaling and high pass scaling respectively, where $\alpha$ and $\beta$ are the frequency domain scaling parameters. They either improve (or) decrease the sampling rate of the signal, i.e, the sampling rate of next stage is $\alpha$ (low pass channel) or $\beta$ (high pass channel) times of that of previous stage. To ensure that the wavelet transform will not be excessively redundant and in the meantime the perfect reconstruction of the filter banks is achievable one has to have $0<\alpha<1,0<\beta \leq 1, \alpha+\beta>1$.

In Figure 1, the sub-band signal $v_{0}(n)$ has a sampling rate of $\alpha f_{s}$ where $f_{s}$ is the sampling rate of the input signal $x(n)$.Likewise the sub-band signal $v_{1}(n)$ has a sampling rate of $\beta f_{S}$

a)

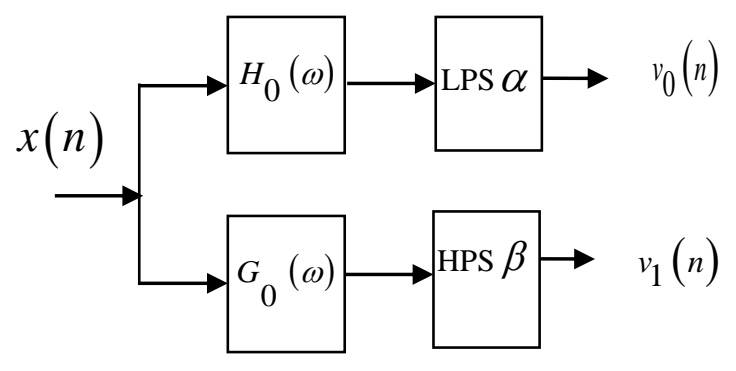


b)

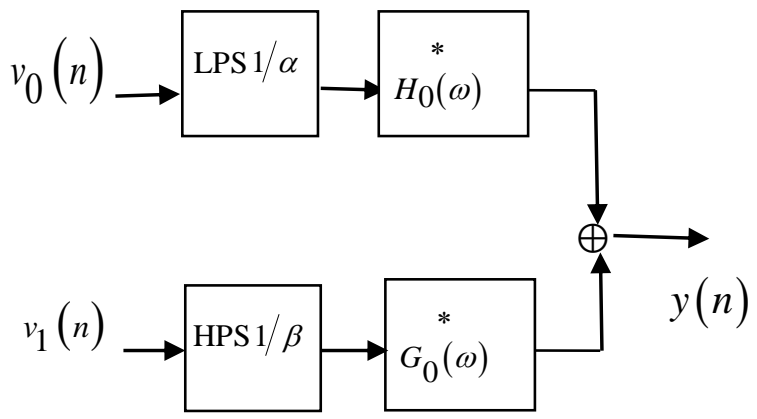

\section{Figure 1. The Single Level TQWT based (a) Decomposition Filter Bank} (b) Reconstruction Filter Bank

\subsection{Multi-Stage TQWT}

Like other kinds of Wavelet Transform, the TQWT is also based on the multi- rate filter banks, as shown in Figure 2. Where, TQWT is implemented through iteratively applying two-channel filter bank, on its low pass channel. Where the low-pass output of each filter bank is the input to the next filter bank. A sub-band is then defined as the output signal of each high pass filter. Considering $J$ the number filter banks, there will be $J+1$ sub-bands, i.e, $\mathrm{J}$ sub bands coming from the high-pass filter output signal of each filter bank and the low-pass filter output signal of the final filter bank. The wavelet coefficients of Sub band signals are denoted by $\left\{d_{j}(n) ; j=1,2, \ldots . ., J\right\}$. Where $\mathrm{j}$ denotes the level of decomposition [10].

The equivalent system for jth level TQWT based decomposition of input signal $x(n)$ to generate the low-pass sub-band signal $a_{j}(n)$ and the high-pass sub-band signal $d_{j}(n)$ is shown in Figure 3.The equivalent frequency response for low-pass and high pass subband signals generated after j-level is given by $H_{j}(\omega)$ and $G_{j}(\omega)$ respectively which are defined in[4]. For multi-stage TQWT, the equivalent sub band filters $H_{j}(\omega)$ and $G_{j}(\omega)$ expressed as

$H_{j}(\omega)=\left\{\begin{array}{l}\prod_{m=0}^{j-1} H_{0}\left(\omega / \alpha^{m}\right), \text { for }|\omega| \leq \alpha^{j} \pi \\ 0, \text { for } \alpha^{j} \pi \leq|\omega| \leq \pi\end{array}\right.$

$$
G_{j}(\omega)=\left\{\begin{array}{l}
G_{0}\left(\omega / \alpha^{j-1}\right) \prod_{m=0}^{j-2} H_{0}\left(\omega / \alpha^{m}\right), \\
\text { for }(1-\beta) \alpha^{j-1} \pi \leq|\omega| \leq \alpha^{j-1} \pi \\
0, \text { forother } \omega \in[-\pi, \pi]
\end{array}\right.
$$


a)

$x(n)$

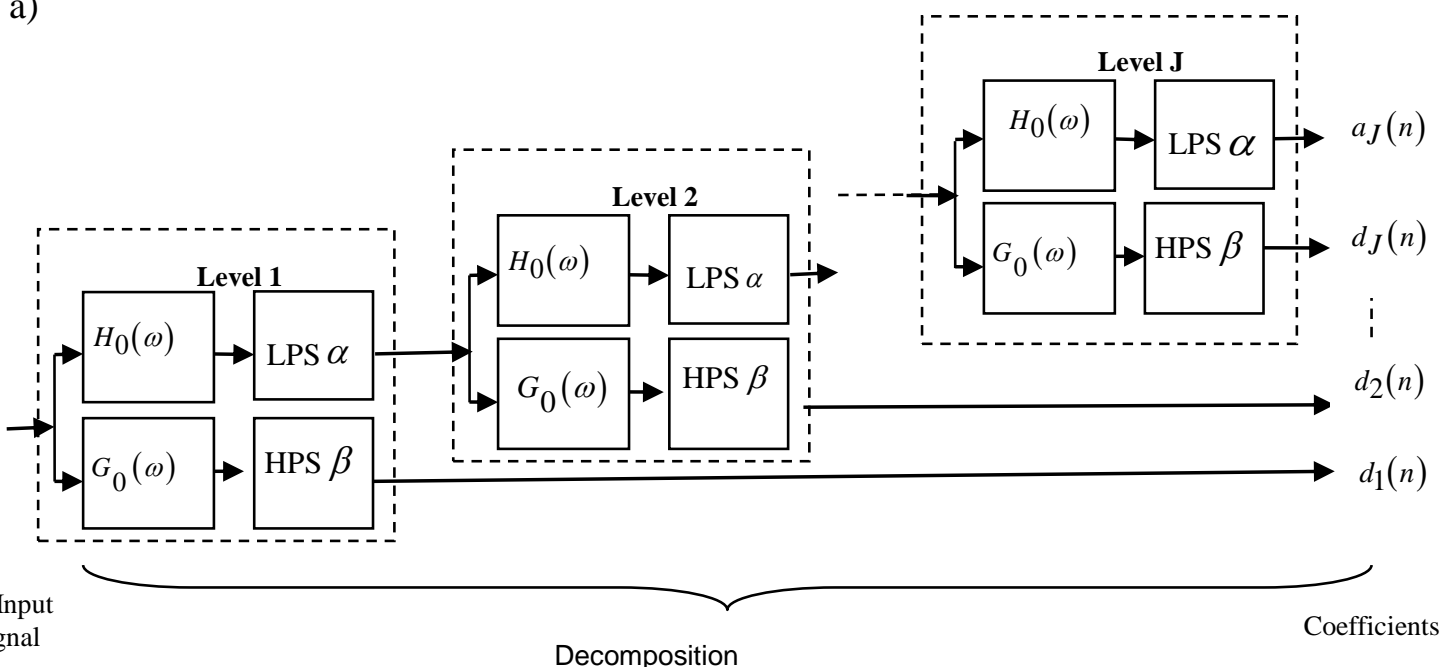

b)

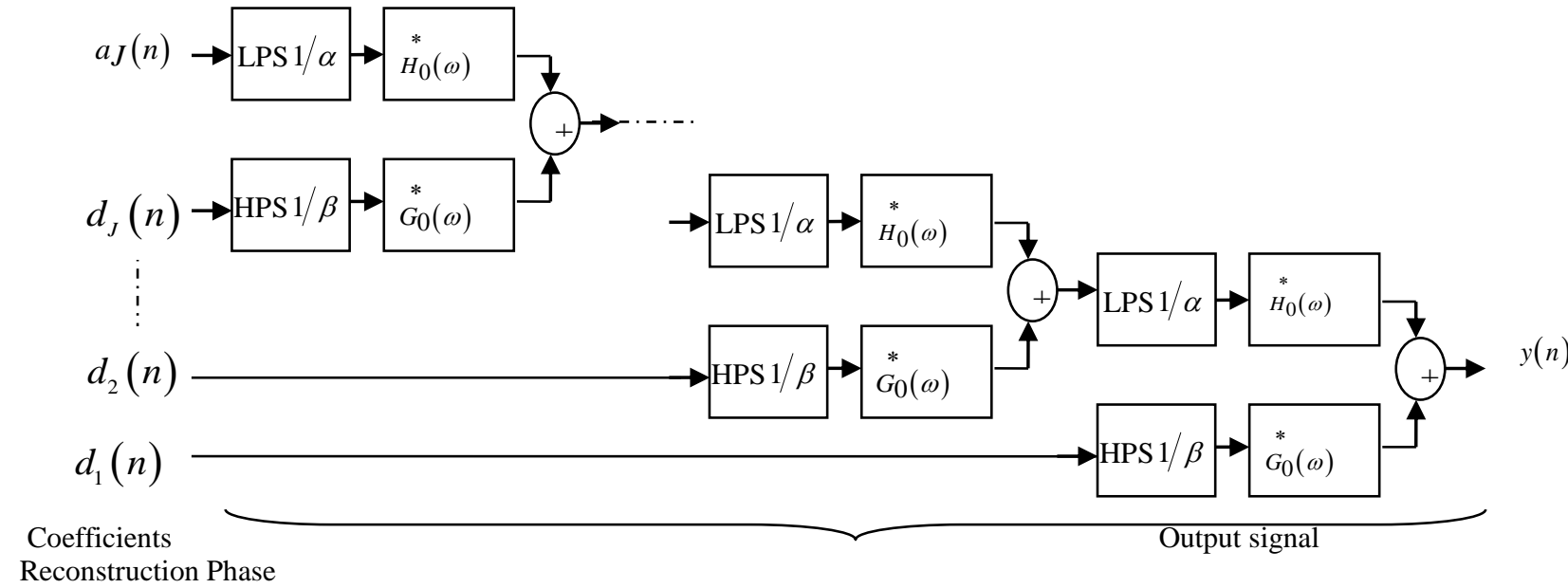

Figure 2. a) The Analysis Filter Bank b) Synthes is Filter Banks for MultiStage TQWT

a) $\quad x(n) \rightarrow H_{j}(\omega) \rightarrow \operatorname{LPS} \alpha^{j} \rightarrow a_{j}(n)$

b)

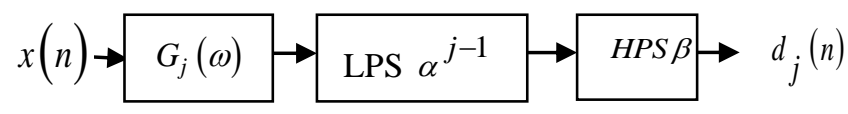

Figure 3. The Equivalent System for jth Level TQWT based Decomposition of Input Signal $x(n)$ to Generate (a) the Low-Pass Sub Band Signal $a_{j}(n)$ and (b) the High-Pass Sub-Band Signal $d_{j}(n)$ 
The equivalent system for the analysis and synthesis filter banks for Multi-stage TQWT is shown in Figure 4.

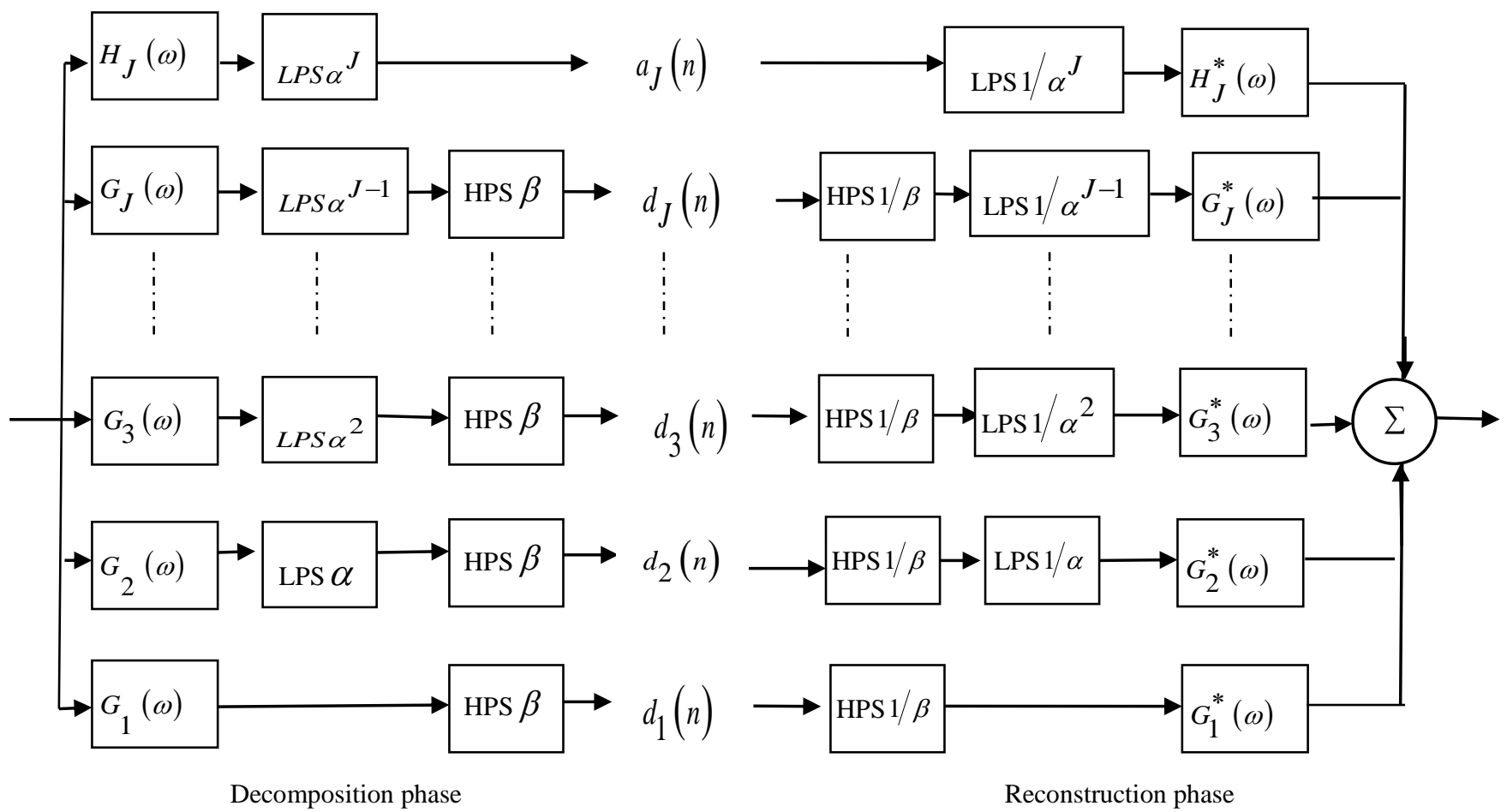

Figure 4. The Equivalent System for the Analysis and Synthesis Filter Banks for Multi-Stage TQWT

\subsection{The Parameters of TQWT}

The main parameters of the TQWT are the Q-factor, which controls the shape of the wavelet, the redundancy $\mathrm{r}$, which localizes the required information, the number of stages (or Decomposition levels) J, which controls the frequency content.

\section{1) Q-factor:}

Generally, Q is a measure of the number of oscillations the wavelet exhibits. If the sampling rate of input signal is $f_{S}$ then at some level of decomposition say, $\mathrm{j}$, the $\mathrm{Q}$ for TQWT can be defined as the ratio of Center frequency $f_{C}(j)$ to the band width $B W(j)$ as follows [11]

$Q=\frac{f_{c}(j)}{B W(j)}$

Where

$$
\begin{aligned}
& f_{C}(j)=\alpha^{j} \frac{2-\beta}{4 \alpha} f_{S}, \text { for } j=1,2, \ldots \ldots, J \\
& B W(j)=\frac{\beta \alpha^{j-1} \pi}{2}
\end{aligned}
$$

From the above equations, we know the both the centre frequency and the band width decrease at the same rate with increase of $\mathrm{j}$.The $\mathrm{Q}$-factor is related to the high-pass scaling 
parameter $\beta$ in the context of the present TQWT implementation, the Q-factor is theoretically defined as

$Q=\frac{2-\beta}{\beta}$

As it reflects the oscillatory behavior of the wavelet, the Q-factor can be set to fit the nature of the signal to which it is applied. In other words the parameter Q can be used to tune the wavelet function to the signal it seeks to model.

For analysis of oscillatory signals, the value of $\mathrm{Q}$ is specified higher such that the underlying wavelets consist of more oscillations with narrower frequency responses relative to their center frequencies. Therefore; it requires more levels of decompositions to cover the spectrum of the signal under study. The property of high Q makes TQWT suitable for effective sparse representation and processing of oscillatory signals. For analysis of piecewise smooth signals like transients, Q is specified lower such that the underlying wavelets consist of fewer oscillations with wider frequency responses relative to their center frequencies that necessitate relatively fewer levels to cover the spectral content of the signal under study. The property of low Q makes TQWT more suitable for the extraction of the transient components [14].

\section{2) Redundancy Parameter (r):}

The redundancy parameter is the total number of wavelet coefficients divided by the length of the input signal to which the TQWT is applied .If the two channel filter bank is iterated with infinitely many levels, the wavelet transform is over sampled by a factor $r$ which is given by the following equation [6]:

$$
r=\frac{\beta}{(1-\alpha)}
$$

It can be interpreted as a measure of how much spectral overlap exists between adjacent band-pass filters. It controls the excessive ringing in order to localize the wavelet in time without affecting its shape. Increasing $r$, while keeping $Q$ unchanged, has the effect of increasing overlapping in the frequency domain of the band-pass filters constituting the TQWT. The parameter $r$ does not affect the general shape of the wavelet of frequency spectrum (they are controlled by Q). With larger $r$, the number of levels $\mathbf{J}$ should be increased in order to cover the same frequency range because of the increased overlap .The specified value of $r$ must be greater than 1 , and a value of 3 (or) greater is generally recommended [7-15].

\section{3) Maximum Number of Levels $\left(J_{\max }\right)$ :}

The parameter $J$ denotes the number of filter banks. After performing a J-stage TQWT decomposition there will be $J+1$ sub bands: the high-pass filter output signal of each filter bank, $\left\{d_{j}(n) ; j=1,2, \ldots . ., J\right\}$ and the low-pass filter output signal of the final filter bank. $a_{J}(n)$. As the analyzed signal cannot be decomposed in to unlimited number of stages , the maximum number of stages $J_{\max }$ has to be set according to length of the input signal $(N)$ and chosen filter scaling parameters $\alpha$ and $\beta$ and is defined by the following equation: 


$$
J_{\text {max }}=\frac{\log \left(\frac{\beta N}{8}\right)}{\log \left(\frac{1}{\alpha}\right)}
$$

Considering the relationship between $(Q, r)$ and scaling parameters $(\alpha, \beta)$, the relationship between $J$ and $(Q, r)$ can be derived as[12].

$$
J_{\max }=\frac{\log \left(\frac{N}{4(Q+1)}\right)}{\log \left(\frac{Q+1}{Q+1-2 / r}\right)}
$$

\subsection{Reconstruction of Time Domain Signals to all the TQWT Levels}

For each decomposition level $j(0<j \leq J)$, we retain the wavelet coefficients of the present level and set all the approximation coefficients and the wavelet coefficients at other levels to zero. This is then followed by the Inverse TQWT, which leads to $J$ reconstructed time-domain signals. Further, if we consider the approximation coefficients as the wavelet coefficients; this will correspond to the $(J+1)$ st level. Then we repeat the same process, i.e, keep the approximation coefficients and set all the other wavelet coefficients to zero and then we get the $(J+1)$ st reconstructed time-domain signal after the Inverse TQWT.TQWT coefficients are not only sparse but also exhibit a clustering(or)grouping Property [13].

\subsection{TQWT Wavelets and Frequency Responses}

Figure 5, shows the wavelet functions and frequency responses of wavelet sub bands of TQWT for two different sets of TQWT parameters. From Figure 5. Three aspects of information can be summarized as follows [10-14]

a)First, for a certain Q-factor, the waveforms of the wavelet embedded in different sub bands are different, though they are with the same Q-factor, with the increase of the decomposition level, the time duration of the wavelet becomes wider, and the oscillation period of the wavelet increases as shown in Figure 5.b, and Figure 5.d. A specific basis associated with specific $Q$ consists of $\mathbf{J}$ wavelets which are translated and/or scaled versions of each other but all have the same degree of oscillation, due to the same Qfactor.

b) Second, the width of the band-pass filter is determined by the Q-factor. For a low Qfactor, the band-pass filters are quite wide, as shown in Figure 5.a, and relatively few levels are needed to cover the spectral content of the signal of interest. In contrast, for a high Q-factor, each band-pass filter is narrow as shown in Figure 5.c, and hence more levels are needed to cover the spectrum of the signal.

c) Third, the role of the Q-factor can also be easily observed in the shape of the wavelet. For a high Q-factor, the wavelets have more sign changes and consist of more oscillatory cycles as shown in Figure 5.d. This property allows effective sparse representation and processing of oscillatory signals. Mean while, for a low Q-factor, the wavelets have fewer sign changes as shown in Figure 5.b, and consist of fewer oscillatory cycles, which are more suitable for the extraction of transient component.

Note that TQWT is specified directly in the frequency domain and implemented using radix-2 FFTs, which can be more efficient. Moreover, the user can directly specify the Q- 
factor and redundancy $\mathrm{r}$ of the TQWT according to the oscillatory behavior of the signal to which it is applied.

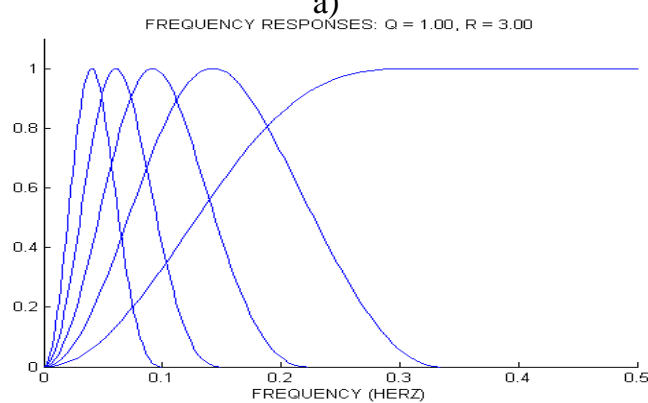

c)

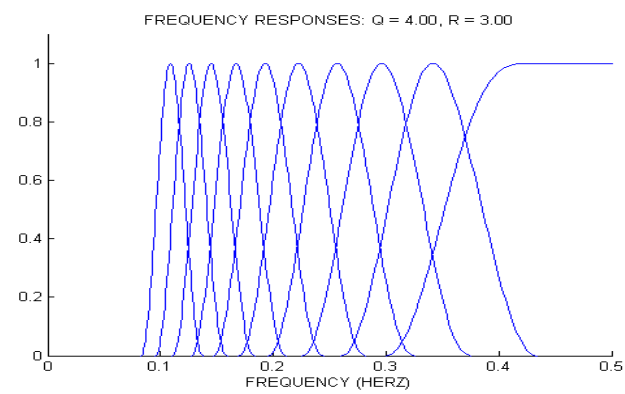

b)

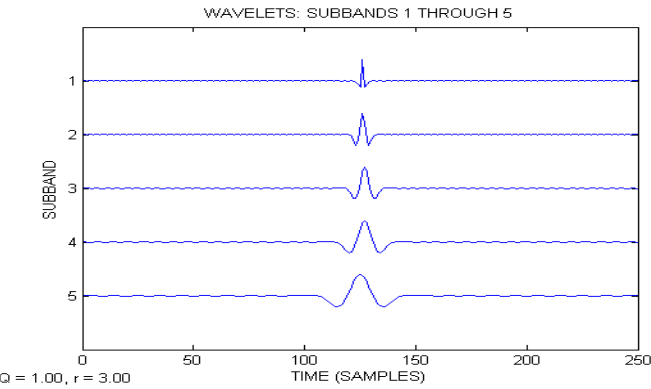

d)

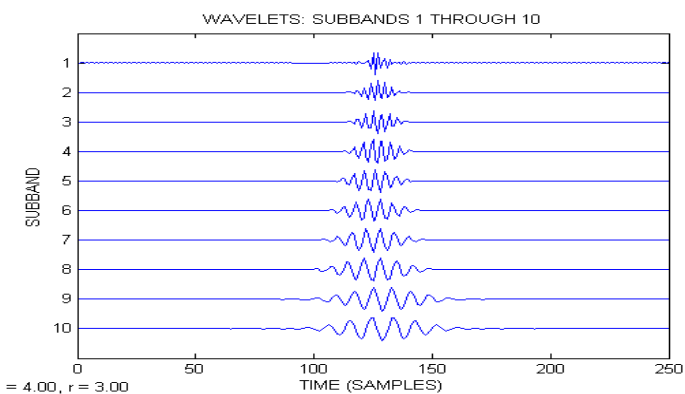

Figure 5. Frequency Responses Spectrum and the Corresponding Time Domain Wavelet Waveform with Different Parameters a) Frequency Responses Spectrum with $Q=1, r=3, J=5$. b) Time Domain Wavelet Waveform of (a) .c) Frequency Responses Spectrum with $Q=4, r=3, J=10 . d$ ) Time Domain Wavelet Waveform of (c)

\section{TVAR Modeling}

\subsection{TVAR Model Review}

A $\mathrm{p}^{\text {th }}$ order Time varying Auto Regressive (TVAR) model representation of a nonstationary signal $x_{n}$, is given by [8].

$$
x_{n}=-\sum_{k=1}^{p} a_{k, n} x_{n-k}+v_{n}
$$

Here $v_{n}$ is zero mean white noise process and $a_{k, n}$ are time-varying coefficients. The time- varying coefficients $a_{k, n}$ are modeled using a linear combination of basis functions. The purpose of the basis functions is to allow time variation of the coefficients in a desired fashion. Denoting $u_{m, n}$ as a basis function and considering a set of $q+1$ functions, the time-variation in the coefficients can be expressed as,

$a_{k, n}=\sum_{m=0}^{q} a_{k, m} u_{m, n}$

TVAR model is then completely specified by the set of parameters $a_{k, m}$ for $\{k=1,2, \ldots, p ; m=0,1, \ldots . q\}$ with $a_{o, m}=1$. The parameters $a_{k m}$ are estimated by minimizing the total squared prediction error given by 


$$
\varepsilon_{p}=\sum_{n=p}^{N-1} \underbrace{\left|x_{n}+\sum_{k=1}^{p} \sum_{m=0}^{q} a_{k m} u_{m n} x_{n-k}\right|^{2}}_{\left|e_{n}\right|}
$$

Here, $\mathrm{N}$ is the length of the data record that is being modeled. Next we define a generalized covariance function $c_{m g}(l, k)$ for $\{l=1,2, \ldots, p ; g=0,1, \ldots ., q\}$ as

$$
c_{m g}(l, k)=\sum_{n=p}^{N-1} u_{m, n} x_{n-k} u_{g, n}^{*} x_{n-l}^{*}
$$

The model parameters $a_{k m}$ that minimize (15) are obtained by solving the following set of generalized covariance equations,

$$
\sum_{k=1}^{p} \sum_{m=0}^{q} a_{k, m} c_{m, g}(l, k)=-c_{0, g}(l, 0)
$$

The above equation represents a system of $p(q+1)$ linear equations, can be expressed in the form of

$C a=-d$

A detailed derivation can be found elsewhere [8].Once the parameters $a_{k, m}$ are determined, we can use (14) to solve for the time-varying coefficients of the TVAR model. The choice of basis functions and order determination using Maximum Likelihood Estimation (MLE) Algorithm can be found elsewhere [9]. In this paper we have chosen Discrete Cosine function as a basis function. This can be expressed as

$u_{m, n}=\alpha(\mathrm{m}) \cos \left(\frac{\pi \mathrm{m}(2 \mathrm{n}+1)}{2 \mathrm{~N}}\right)$

Where

$$
\begin{aligned}
& \alpha(\mathrm{m})=\left\{\begin{array}{l}
\sqrt{\frac{1}{N}} ; \text { for }, m=0 \\
\sqrt{\frac{2}{N}} ; m=1,2 \ldots \ldots . q
\end{array}\right. \\
& \text { and } n=1,2, \ldots . N
\end{aligned}
$$

\subsection{TVAR Based IF Estimation}

1) Compute TVAR model order $p$ and $q$ using MLE Algorithm, choose the basis function $u_{m, n} \mathrm{~m}=1,2 \ldots . . \mathrm{q}, \mathrm{n}=1,2, \ldots \ldots, \mathrm{N}$.

2) Compute $c_{m g}(l, k)$ by means of equation (16) to set up the matrix $C$ in (18), as well, use $c_{m g}(l, k)$ to calculate $d$ in (18).

3) Calculate the TVAR Parameters $a_{k, m}$ by solving $\mathrm{Ca}=-\mathrm{d}$, in (18) and form the coefficients $a_{k, n}$ using (14)

4) Solve the roots of the time-varying autoregressive Polynomial formed by TVAR linear prediction filter $\mathrm{A}(\mathrm{z} ; \mathrm{n})=1+\sum_{k=1}^{p} a_{k, n} z^{-k}$ at each instant $\mathrm{n}$ to find the time-varying 
poles: $P_{i, n}, \mathrm{i}=1,2 \ldots \mathrm{p}$

5) The IF of the signal, for each sample instant $n$ can be estimated from the instantaneous angles of the poles using $f_{i, n}=\frac{\arg \left[P_{i, n}\right]}{2 \pi}$ for $\left|P_{i, n}\right| \cong 1$

\section{Simulation Examples}

Instantaneous frequency estimation of multi component non stationary signal can be estimated based on joint application of the TQWT and TVAR Model. The proposed technique is composed of two steps:

\section{Step1:}

1) Decompose the multi-component signal by using the TQWT into $(\mathrm{J}+1)$ number of wavelet coefficients sub bands.

2) It is observed that the distribution of signal energy with respect to TQWT sub bands has clustering (or) grouping property. Where each group corresponding to one mono-component signal.

3) Applying Inverse TQWT to each sub band results to $(\mathrm{J}+1)$ reconstructed time domain signals.

4) A mono component signal is obtained by adding the reconstructed time domain signals corresponding to each cluster.

\section{Step2:}

TVAR Model is applied to each extracted mono component signal to estimate Instantaneous Frequency of non stationary signal.

To demonstrate the effectiveness of the proposed method here we are considering Multi component Non stationary signals.

\subsection{Multi Component AM-FM Signal}

The sequence $y_{1}(n)$ consisting of $\mathrm{M}$ single-tone AM-FM sinusoidal signals is represented by [2]

$$
y(n)=\sum_{i=1}^{M} A_{i}\left(1+\mu_{i} \cos \left(v_{a i} n\right)\right) \cos \left(\omega_{i} n+\beta_{i} \sin \left(v_{f i}{ }^{n}\right)\right)
$$

For simulation, the signal consisting of two $(M=2)$ single tone AM-FM signals is sampled at $\mathrm{N}=512$ points. Two sets of parameters in (21) are chosen as

$$
\begin{aligned}
& A_{1}=1, \mu_{1}=0.8, v_{a 1}=2 \pi \times 0.0063, \\
& \omega_{1}=2 \pi \times 0.1250, \beta_{1}=2, v_{f 1}=2 \pi \times 0.0094, \\
& A_{2}=1, \mu_{2}=0.6, v_{a 2}=2 \pi \times 0.0125, \\
& \omega_{2}=2 \pi \times 0.2500, \beta_{2}=1.5, v_{f 2}=2 \pi \times 0.0125 .
\end{aligned}
$$

True IF for first component signal is given by 


$$
f_{1 n}=\frac{\omega_{1}+\left(\beta_{1}^{*} v_{f 1}\right) \cos \left(v_{f 1} n\right)}{2 \pi}
$$

True IF for second component signal is given by

$$
f_{2 n}=\frac{\omega_{2}+\left(\beta_{2}{ }^{*} v_{f 2}\right) \cos \left(v_{f 2^{n}}\right)}{2 \pi}
$$

Figure 6, show the multi -component AM-FM signal. TQWT with parameters $\mathrm{Q}=5$, $\mathrm{r}=3, \mathrm{~J}=10$, is applied for multi-component AM-FM signal. TQWT decompose the AMFM signal into 11 sub bands as shown in Figure 7. Distribution of signal energy with respect to TQWT sub band shown in Figure 8. Note that in Figure7.and Figure8. Sub band 1 corresponds to high -pass sub band, while sub band 11 corresponds to the lowpass sub band.

From Figure 8, We observe that sub bands 10 to 11 form one cluster and sub bands 4 to 6 form another cluster, where each cluster corresponds to a mono-component signal. Two clusters in Figure 8, implies the original signal is a two component signal. Note that the distribution of signal energy in remaining sub bands are very less $(<1 \%$ of total signal energy).For each sub band level $j(0<j \leq 11)$, we retain the wavelet coefficients of the present level and set all the approximation coefficients and the wavelet coefficients of other levels to zero. This is then followed by the Inverse TQWT, which leads to 11 reconstructed time-domain signals. By adding 4-6 reconstructed time-domain signals we can estimate one mono-component signal. By adding 10-11 reconstructed time-domain signals we can estimate another mono-component signal. Figure 9 show the True and Estimated First mono component signal. Figure 10 show the True and Estimated second mono component signal

TVAR Model with Discrete Cosine basis is applied to each extracted mono component signal to find IF of each mono-component signal. For First mono component signal TVAR Model order $\mathrm{p}=2$, and $\mathrm{q}=22$, for Second mono component signal TVAR Model order $\mathrm{p}=2$, and $\mathrm{q}=28$, are estimated using MLE Algorithm. Estimated TVAR Model parameters $a_{1, n}, a_{2, n}$, for first mono- component signal and second mono component signals are shown in Figure 11, and Figure 14, respectively. Time varying poles trajectory of the first mono-component and second mono-component signals are shown in Figure 12, and Figure 15, respectively. True and estimated IF using TVAR Model of the first mono component signal and second mono component signal are shown in Figure 13, and Figure 16, respectively. From Figure 13, and Figure 16, we observe that estimated IF is very close to true IF.

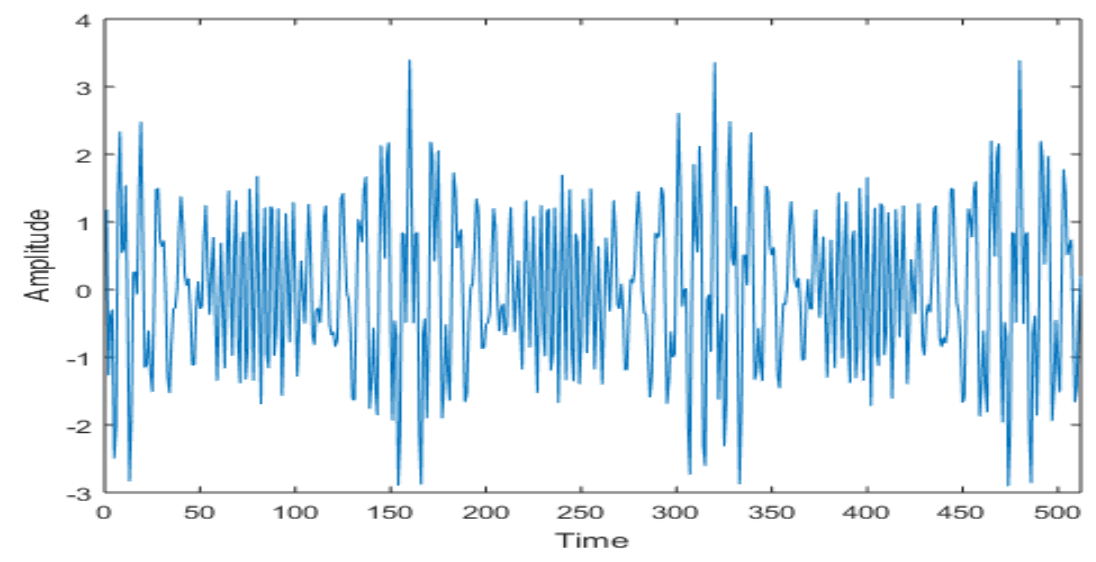

Figure 6. Multi- Component AM-FM Signal 


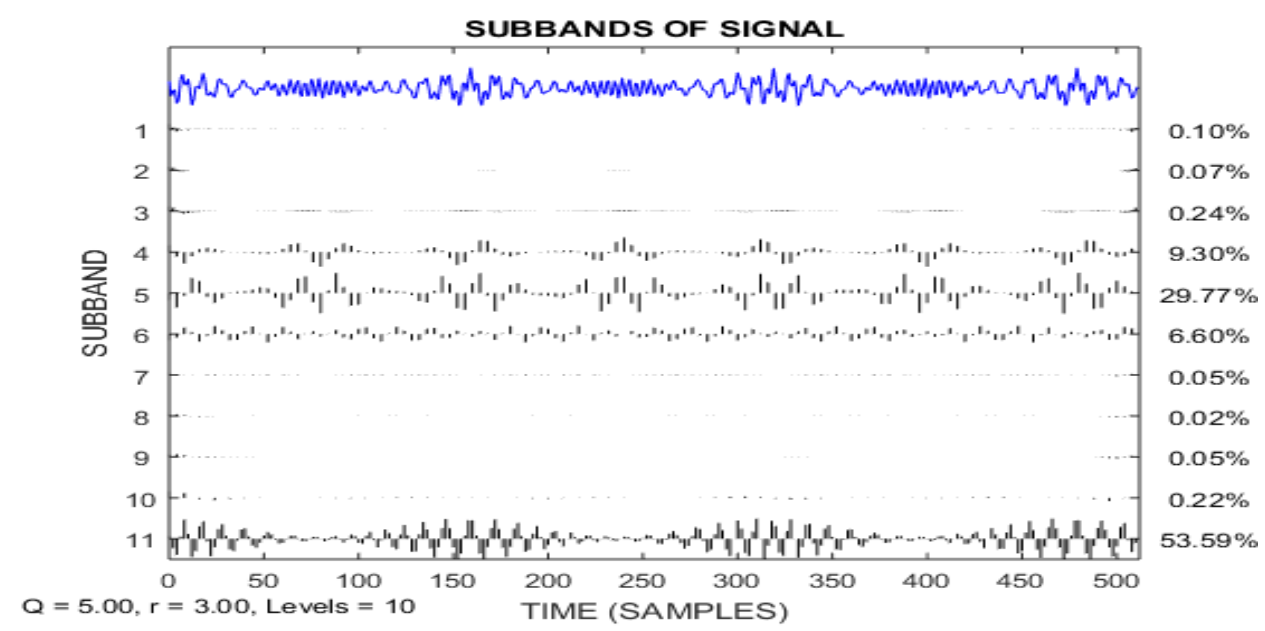

Figure 7. Sub Bands of Signal

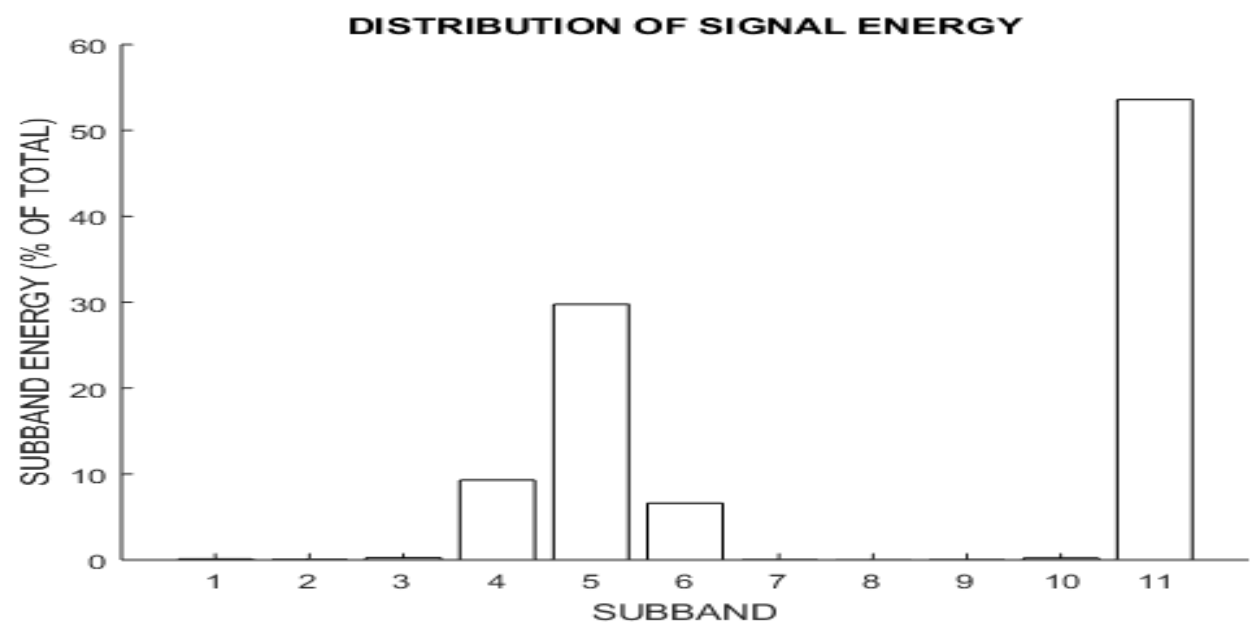

Figure 8. Distribution of Signal Energy

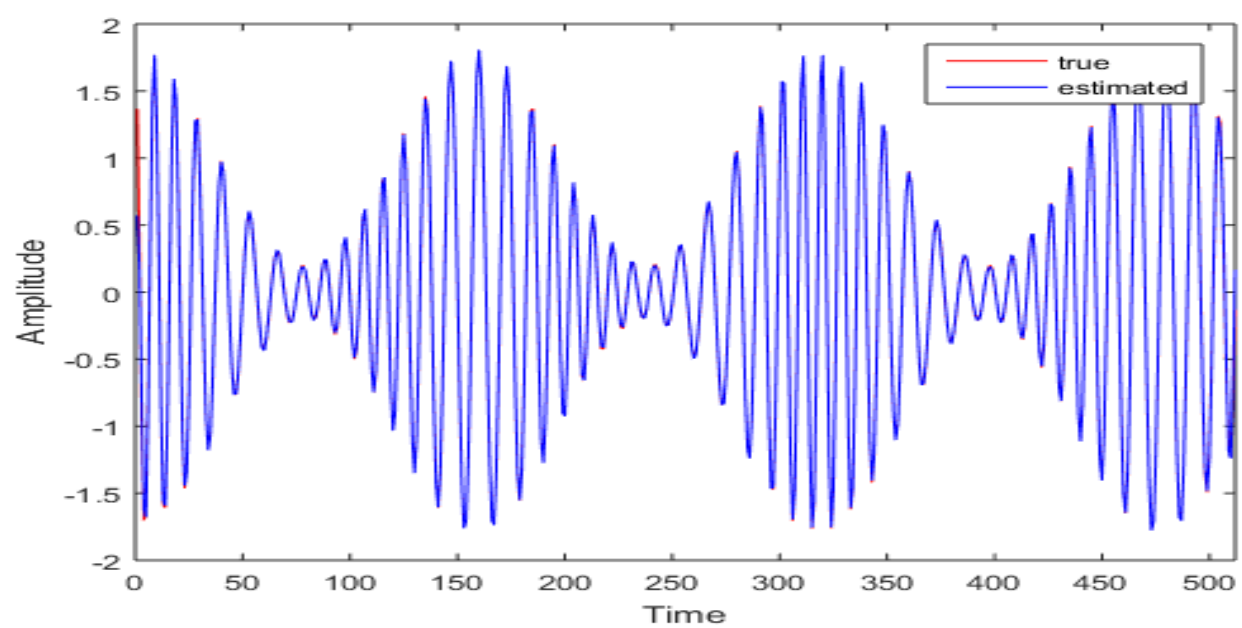

Figure 9. True and Estimated Second Mono- Component Signal 


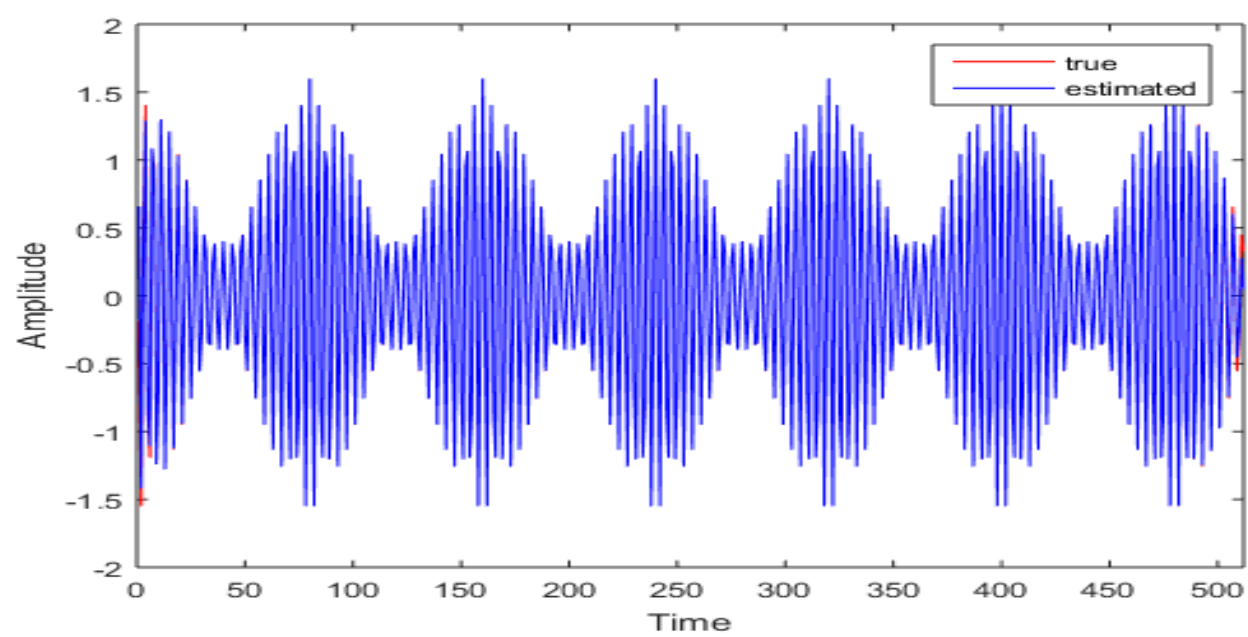

Figure 10. True and Estimated Second Mono- Component Signal
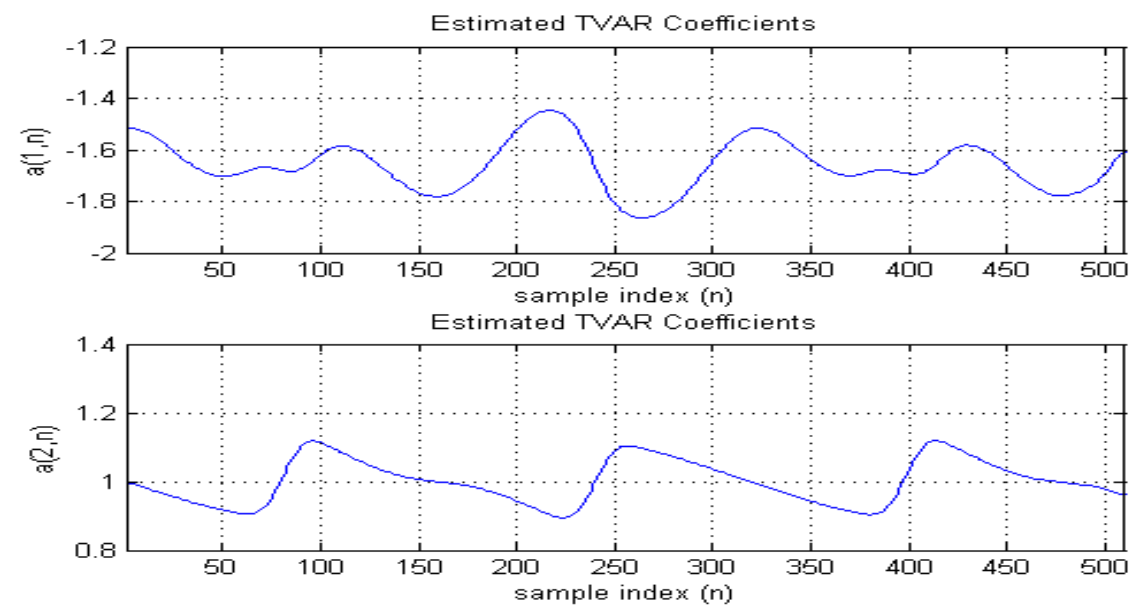

Figure 11. Estimated TVAR Coefficients for First Mono-Component Signal

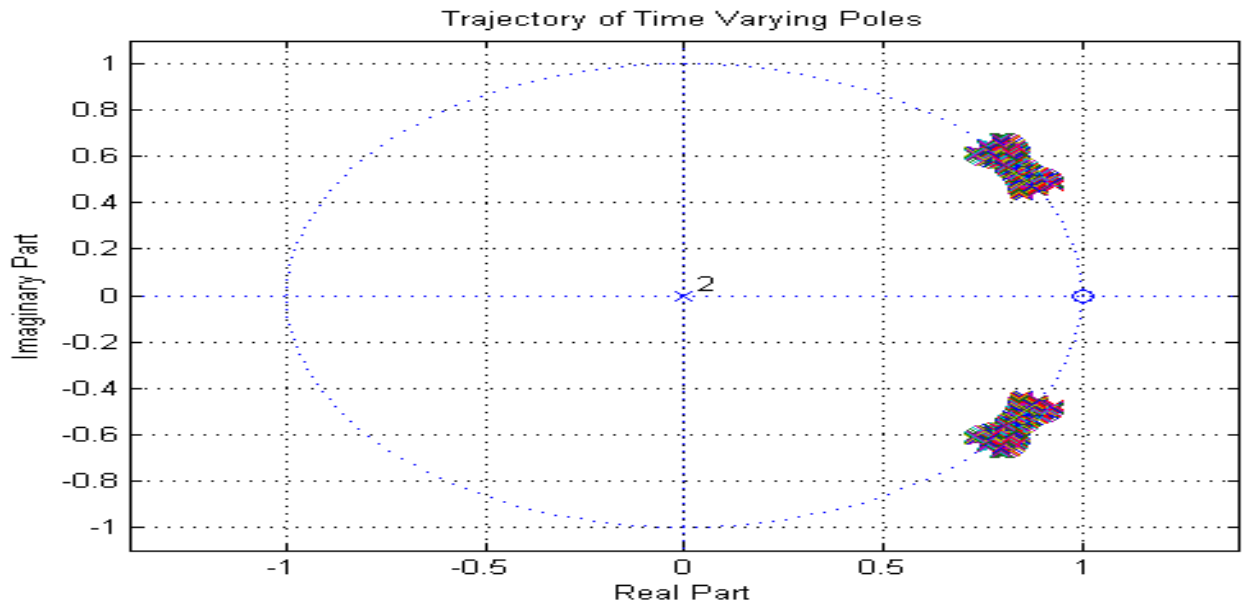

Figure 12. Trajectory of Time Varying Poles for First Mono-Component Signal 


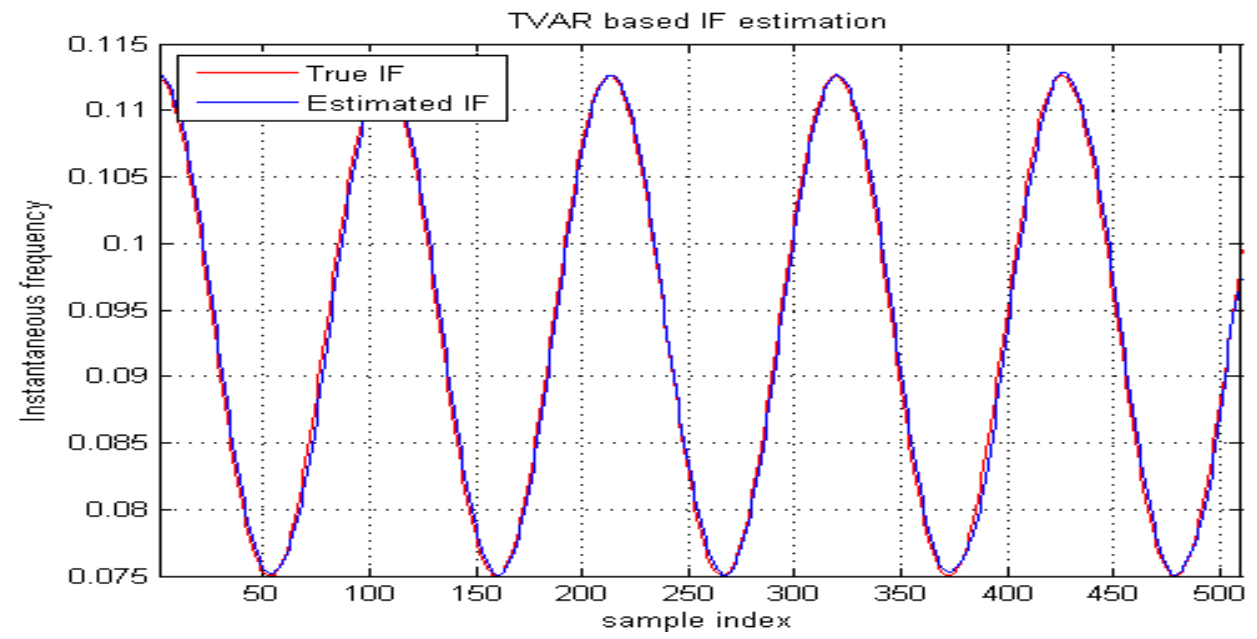

Figure 13. TVAR based IF Estimation for First Mono-Component Signal
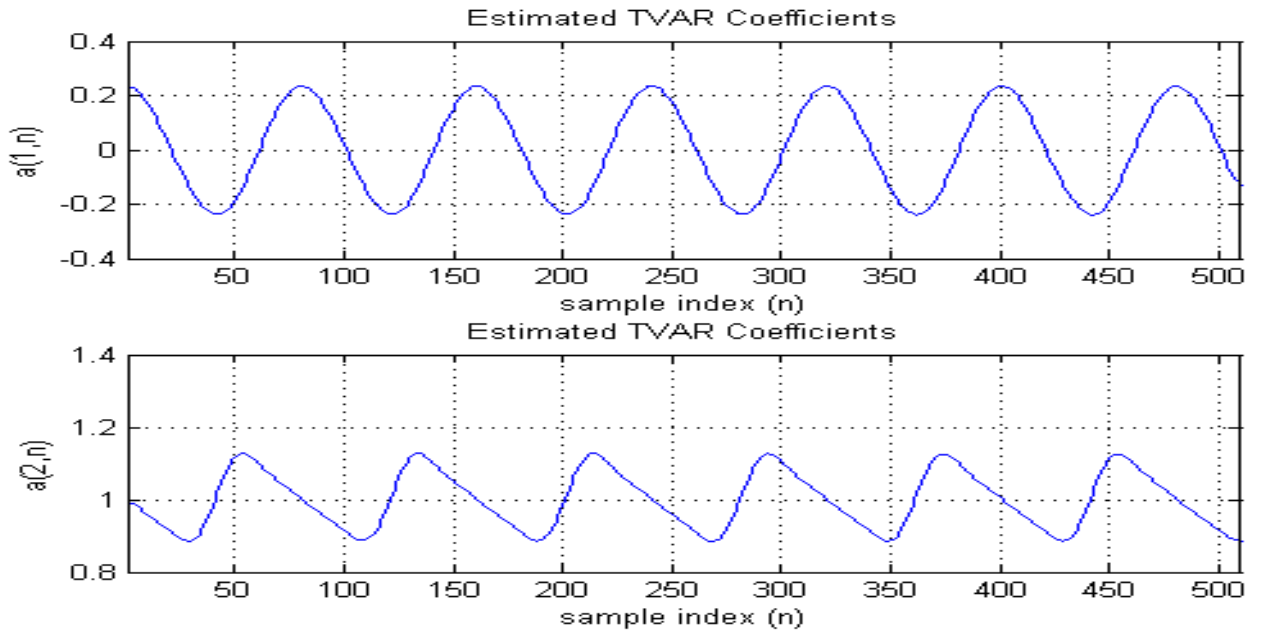

Figure 14. Estimated TVAR Coefficients for Second Mono-Component Signal

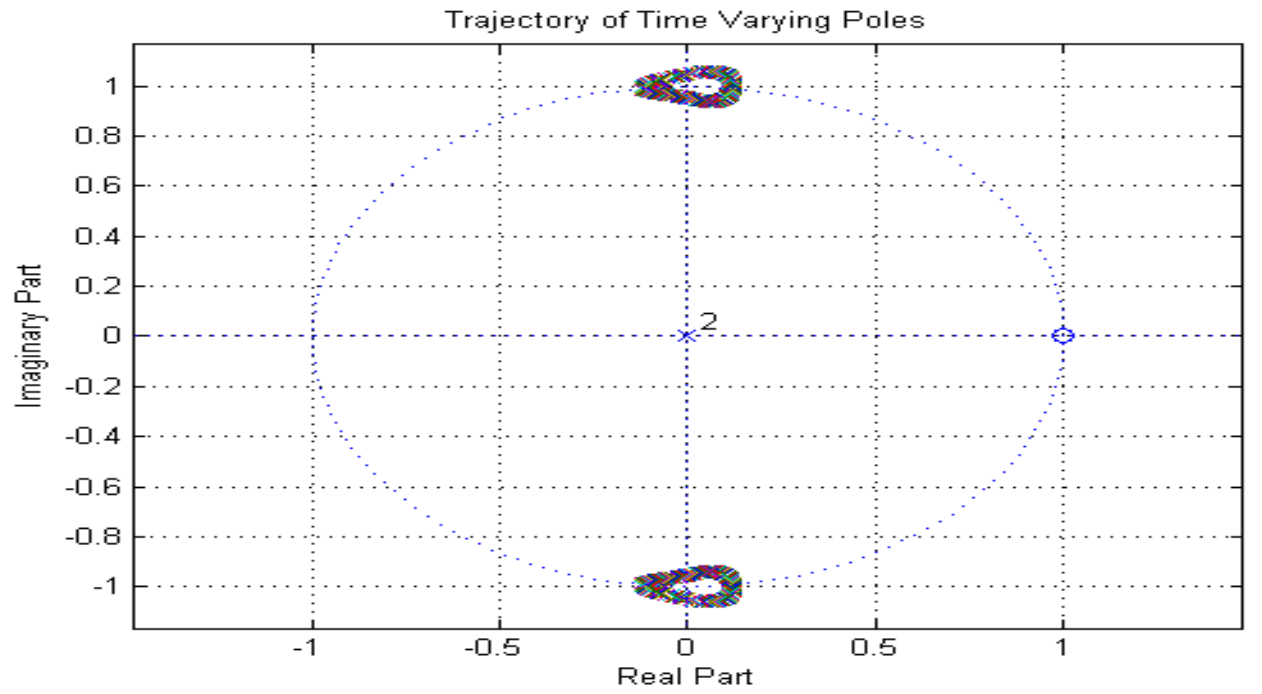

Figure 15. Trajectory of Time Varying Poles for Second Mono-Component Signal 


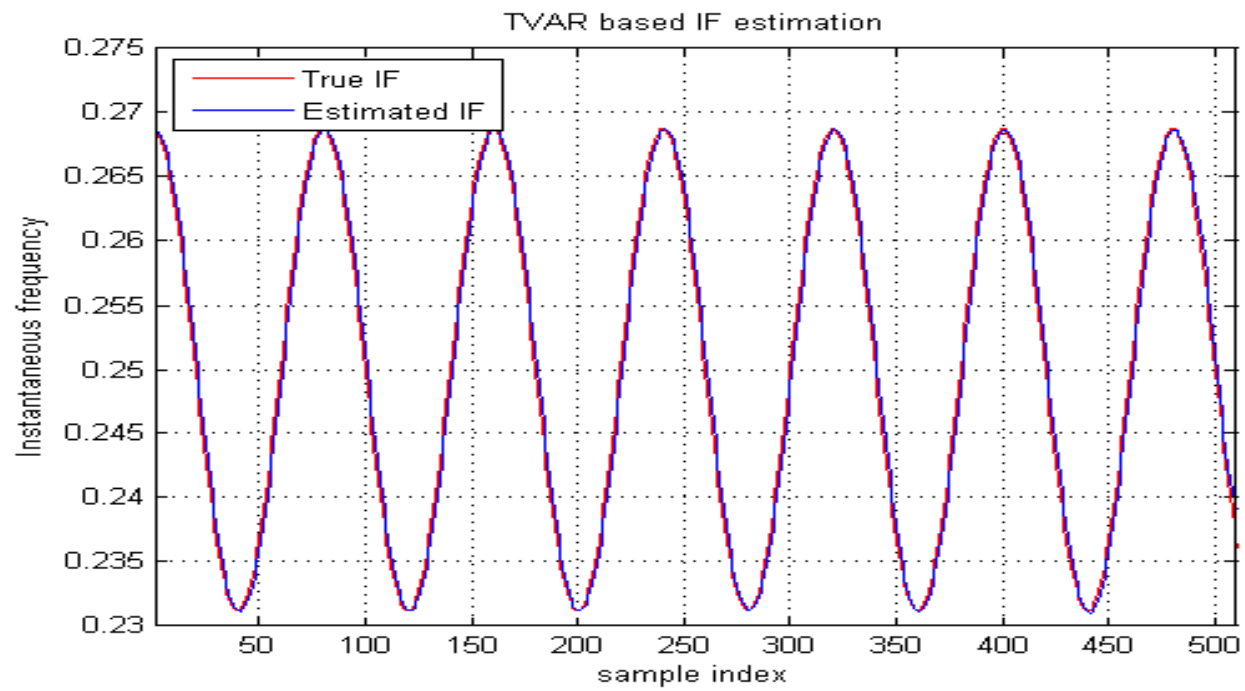

Figure 16. TVAR based IF Estimation for Second Mono-Component Signal

\subsection{Multi-Component Non-Stationary Signal}

The signal $y_{2}(n)$ is a two-component non-stationary signal. One component is linear FM whereas the other component is non-linear FM chirp. The mathematical expression for the signal $y_{2}(n)$ can be given in discrete time-domain for $\mathrm{n}=1,2 \ldots, 500$. as follows:

$$
\begin{aligned}
& y_{2}(n)=x_{1}(n)+x_{2}(n) \\
& x_{1}(n)=\cos [(\pi n / 1000+22) n / 10] \\
& x_{2}(n)=\cos [(3 \pi n / 500+471) n / 500+30 \cos (\pi n / 256)]
\end{aligned}
$$

True IF for first component signal is given by

$$
f_{1 n}=\frac{1}{2 \pi}\left(\frac{2 \pi n}{10000}+\frac{22}{100}\right)
$$

True IF for second component signal is given by

$$
f_{2 n}=\frac{1}{2 \pi}\left(\frac{6 \pi n}{25000}+\left(\frac{471}{500}\right)-\left(\frac{30 \pi}{256}\right) \sin \left(\frac{\pi n}{256}\right)\right)
$$

Figure 17, show the multi-component non-stationary signal,TQWT with parameters $\mathrm{Q}=5, \mathrm{r}=1.55, \mathrm{~J}=8$, is applied for signal $y_{2}(n)$. TQWT decompose the signal into $9 \mathrm{sub}$ bands as shown in Figure 18. Distribution of signal energy with respect to sub bands shown in Figure 19, Note that in Figure 18, and Figure 19, Sub band 1 corresponds to high -pass sub band, while sub band 9 corresponds to the low-pass sub band.

From Figure 19, We observe that first 2 sub bands form one cluster and sub bands 4-9 form another cluster, where each cluster corresponds to a mono-component signal. Two clusters in Figure 19, implies the original signal is a two component signal. Note that the distribution of signal energy in remaining sub bands are very less $(<1 \%$ of total signal energy).For each sub band level $j(0<j \leq 8)$, we retain the wavelet coefficients of the present level and set all the approximation coefficients and the wavelet coefficients of other levels 
to zero. This is then followed by the Inverse TQWT, which leads to 9 reconstructed timedomain signals. By adding first 2 reconstructed time-domain signals we can estimate one mono-component signal. By adding 4-9 reconstructed time-domain signals we can estimate another mono-component signal. Figure 20, shows the True and Estimated First mono component signal.Figure21.shows the True and Estimated Second monocomponent signal.

TVAR Model with Discrete Cosine basis is applied to each mono-component signal to find IF of each mono-component signal. For First mono-component signal, TVAR Model order $\mathrm{p}=2$, and $\mathrm{q}=32$, for Second mono-component signal TVAR Model order $\mathrm{p}=2$, and $\mathrm{q}=48$, are estimated using Maximum Likely hood estimation Algorithm. Estimated TVAR Model parameters $a_{1, n}, a_{2, n}$, for first mono-component signal and second mono-component signals are shown in Figure 22, and Figure 25, respectively. Time varying poles trajectory of the first mono-component and second mono-component are shown in Figure 23, and Figure 26, respectively.True and Estimated IF using TVAR Model of the first mono-component signal and second mono-component signal are shown in Figure 24, and Figure 27, respectively. From Figure 24, and Figure 27, we observe that estimated IF is close to true IF.

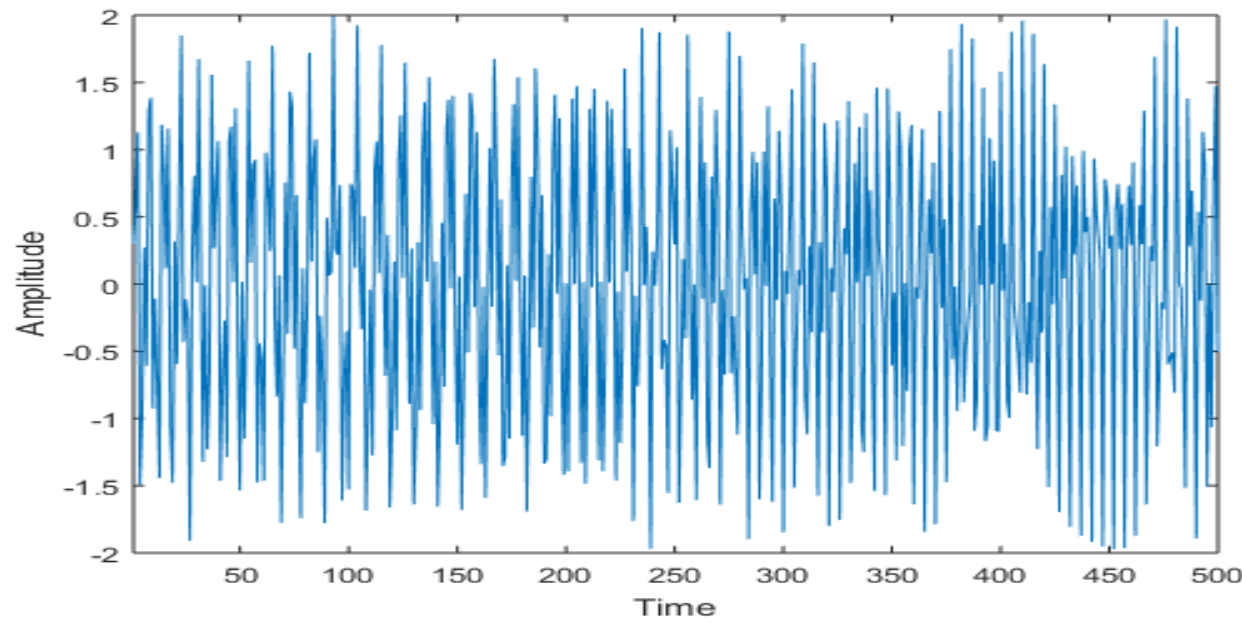

Figure 17. Multi-Component Signal with a LFM Chirp and a Non-Linear FM Chirp

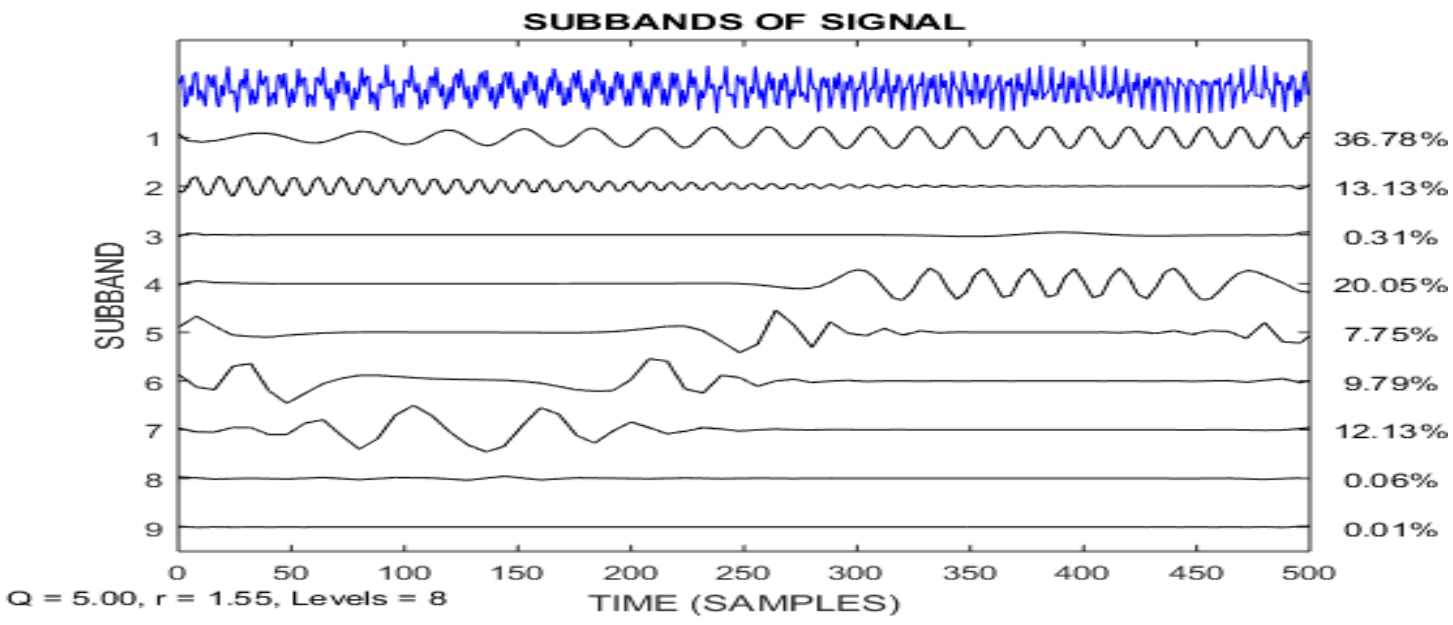

Figure 18. Subbands of a Signal 


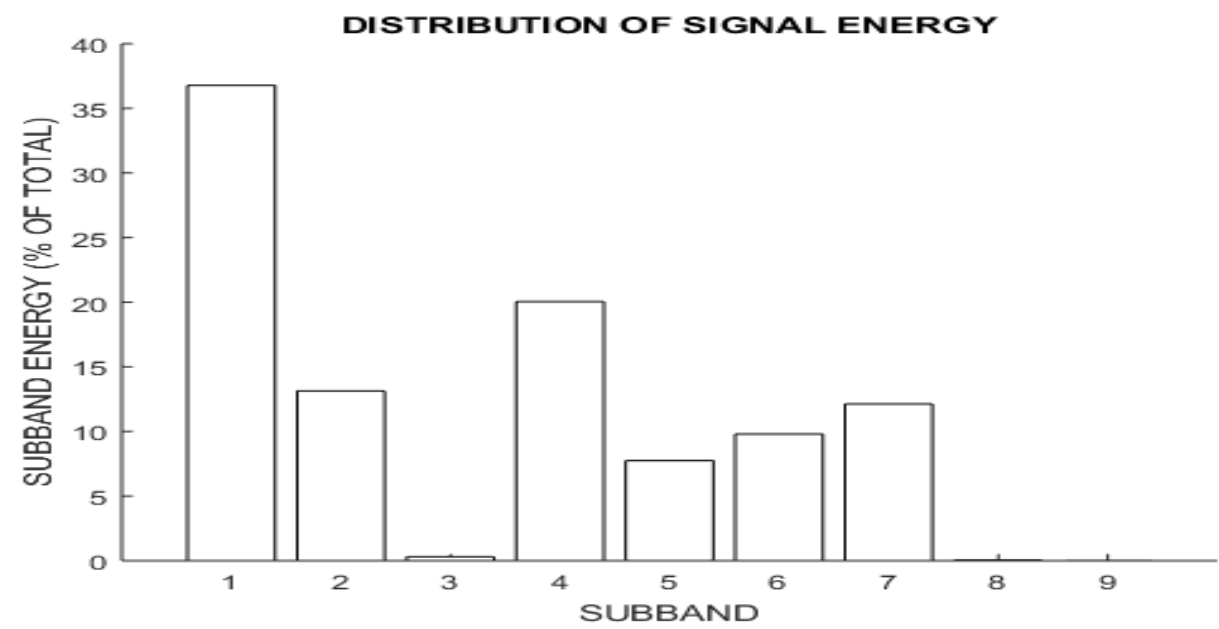

Figure 19. Distribution of Signal Energy with Respect to Subband

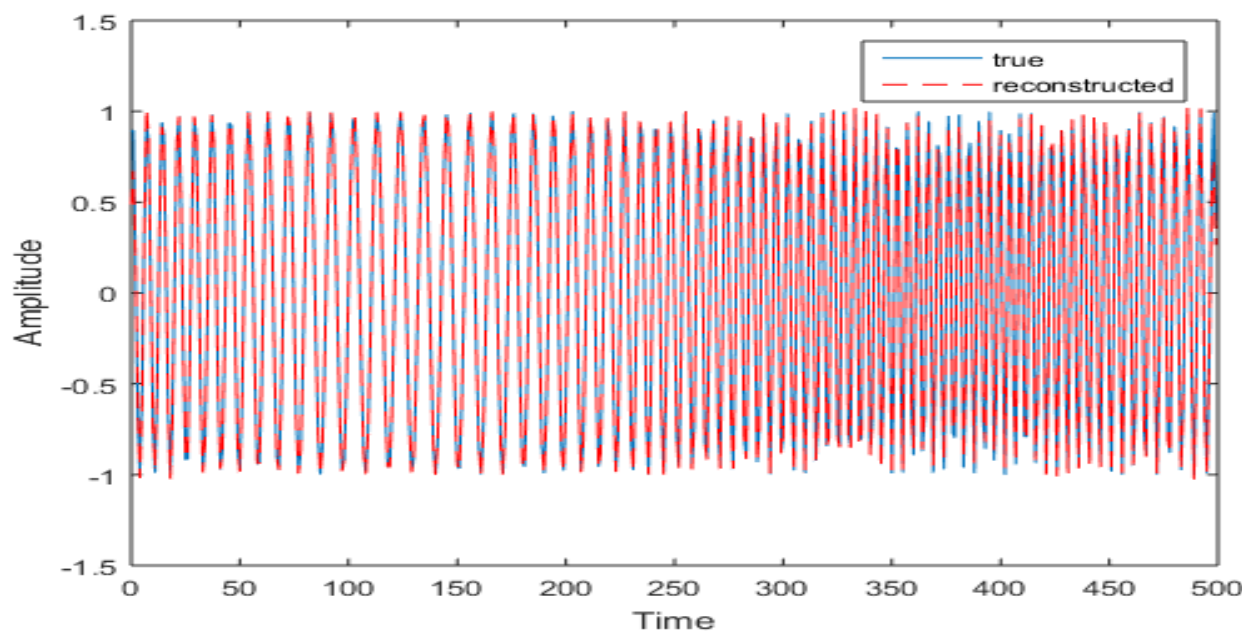

Figure 20.True and Reconstructed First Mono- Component Signal

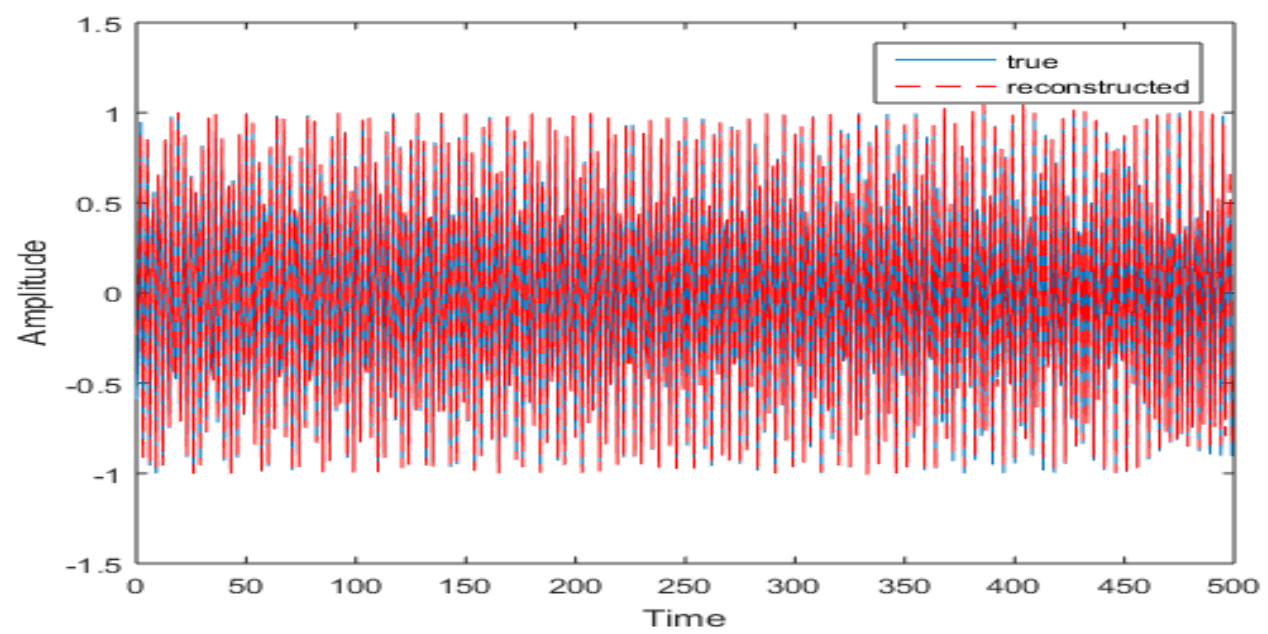

Figure 21.True and Reconstructed Second Mono- Component Signal True IF 

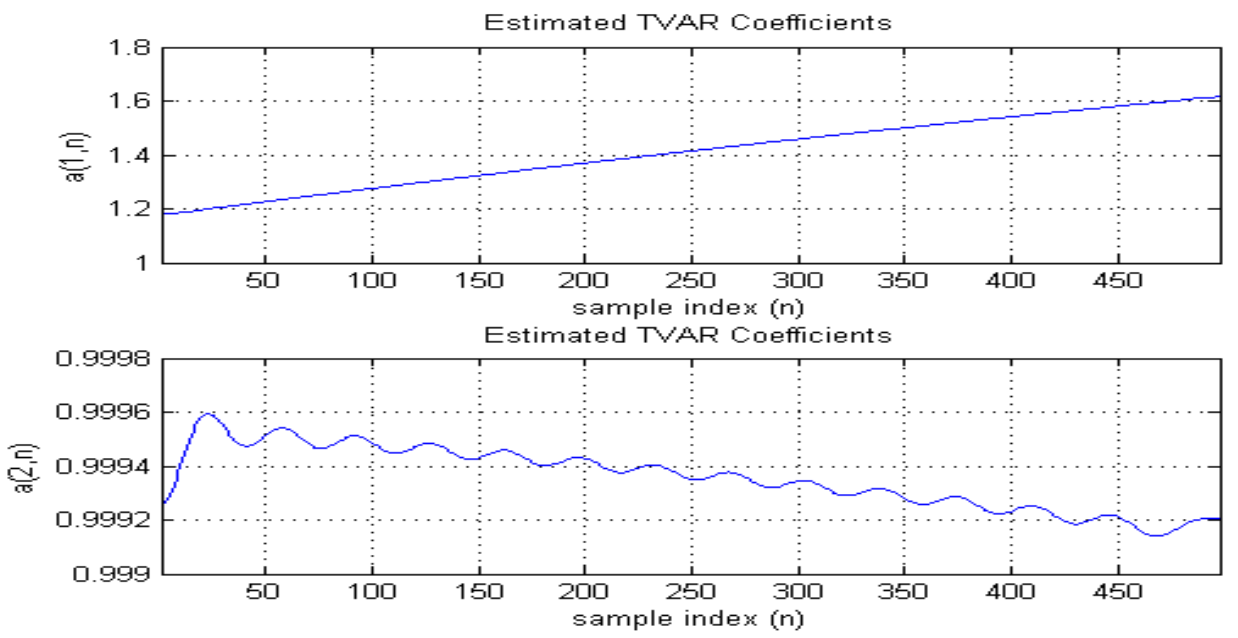

Figure 22. Estimated TVAR Coefficients for First Mono- Component Signal

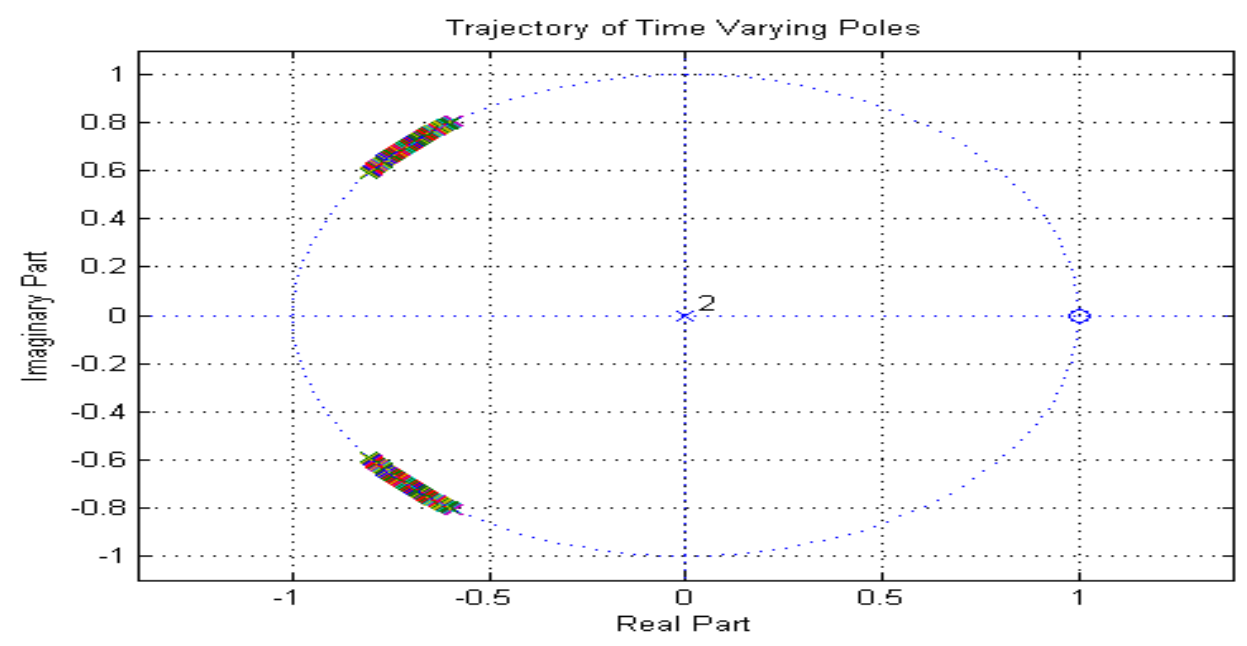

Figure 23. Trajectory of Time Varying Poles for First Mono-Component Signal

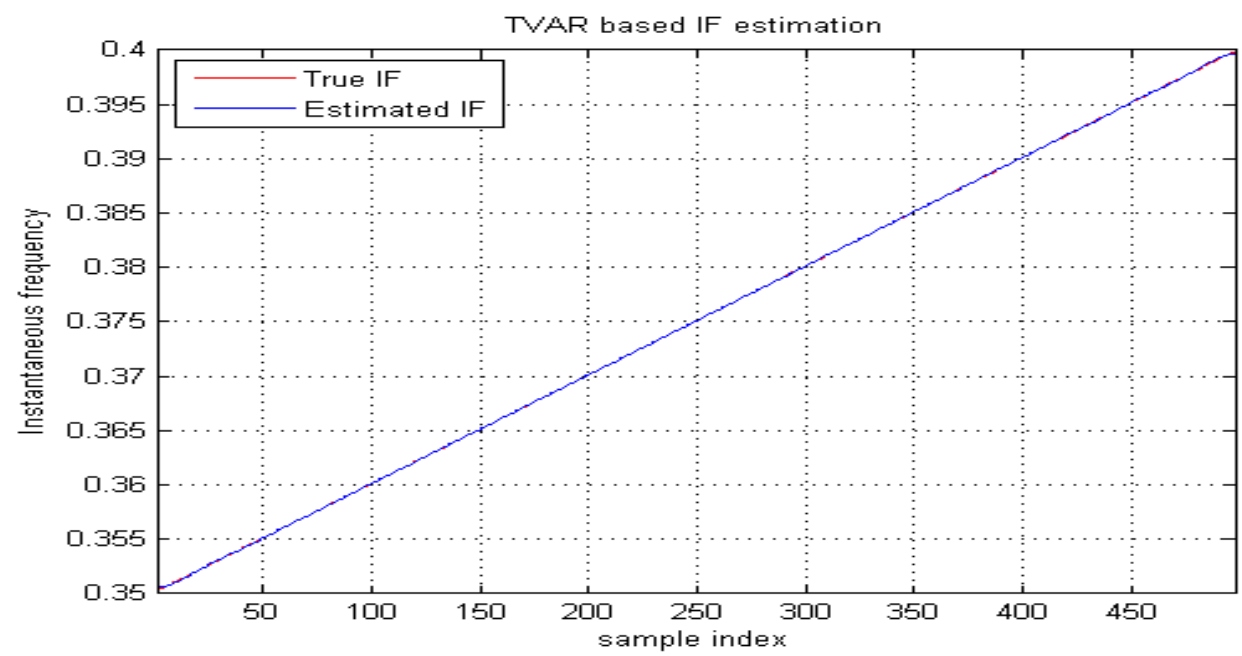

Figure 24. TVAR based IF Estimation for First Mono- Component Signal 

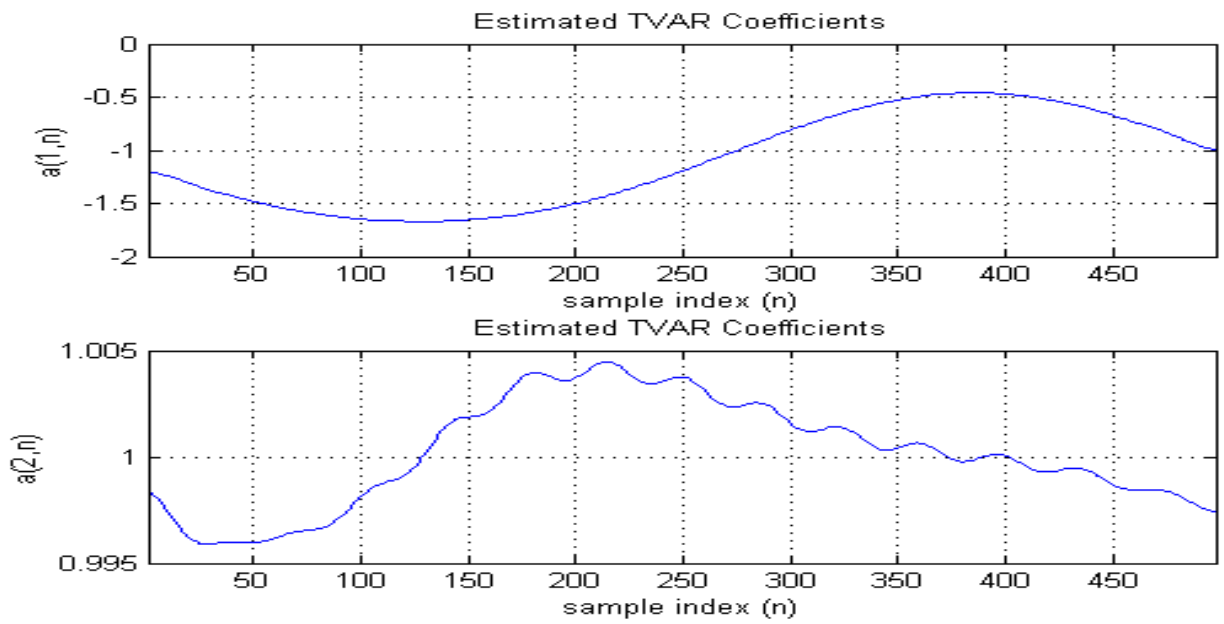

Figure 25. Estimated TVAR Coefficients for Second Mono- Component Signal

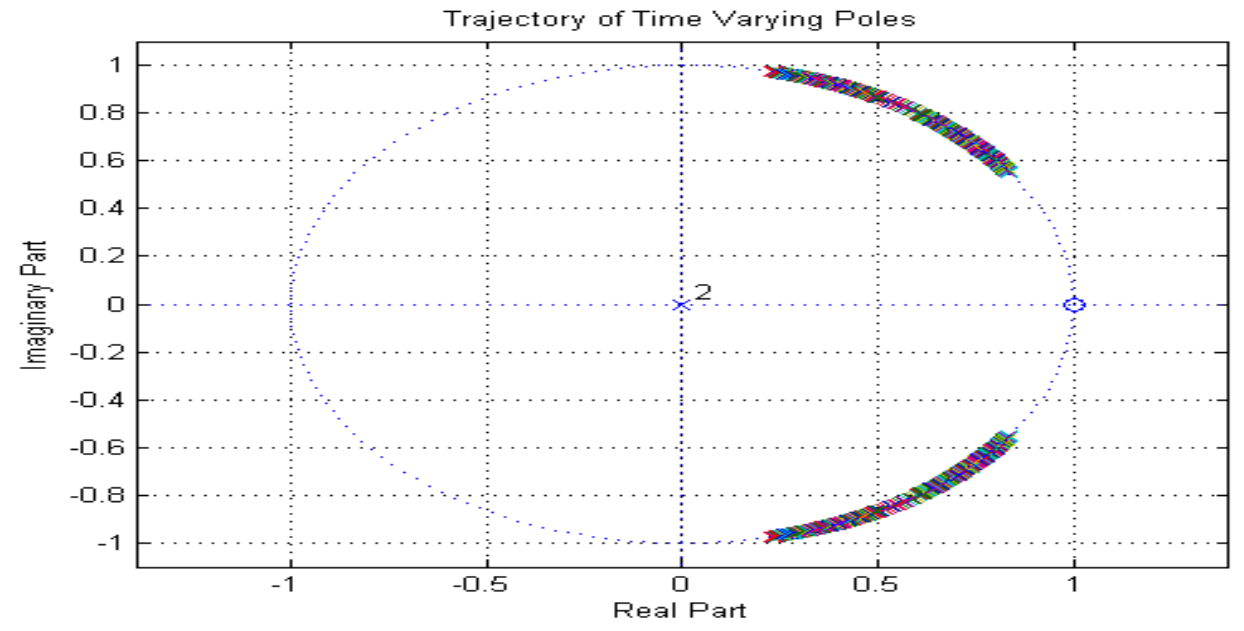

Figure 26. Trajectory of Time Varying Poles for Second Mono-Component Signal

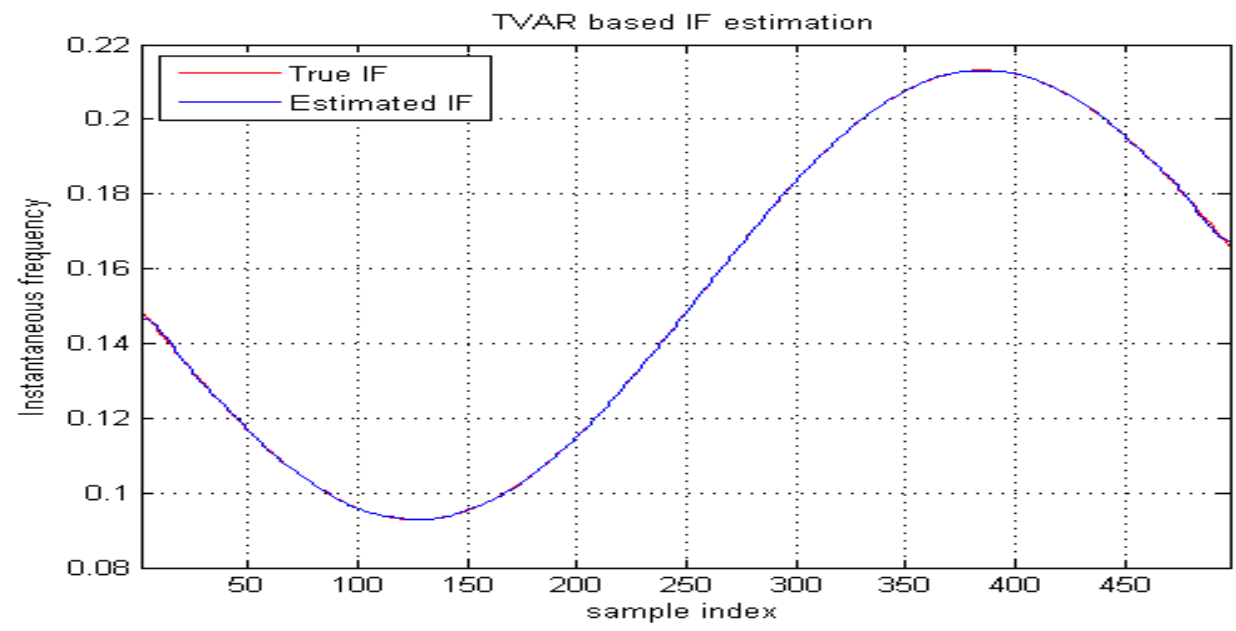

Figure 27. TVAR based IF Estimation for Second Mono-Component Signal 


\section{Conclusion}

A new TQWT decomposition and reconstruction approach for decomposing a multicomponent non-stationary signal in to mono-component signals has been proposed in this paper. The proposed approach is based on distribution of signal energy with respect sub band has clustering property for mono-component signals. For each extracted mono component signal Time varying Auto regressive model is applied to estimate the instantaneous frequency.

\section{References}

[1] P. Maragos, J. F. Kaiser and T. F. Quatieri, "Energy separation in signal modulations with application to speech analysis." Signal Processing, IEEE Transactions on, vol. 41, no. 10, (1993), pp. 3024-3051.

[2] R. B. Pachori and Pradip Sircar. "Analysis of multicomponent AM- FM signals using FB-DESA method." Digital Signal Processing, vol. 20, no. 1, (2010), pp. 42-62.

[3] E. H. Norden "The empirical mode decomposition and the Hilbert spectrum for nonlinear and nonstationary time series analysis." Proceedings of the Royal Society of London A: Mathematical, Physical \& Engineering Sciences, The Royal Society, vol. 454, no. 1971, (1998).

[4] I. W. Selesnick, "Wavelet transform with tunable Q-factor." Signal Processing, IEEE Transactions on vol. 59, no. 8, (2011), pp. 3560- 3575.

[5] I. W. Selesnick,. "Resonance-based signal decomposition: A new sparsity-enabled signal analysis method." Signal Processing, vol. 91, no. 12, (2011), pp. 2793-2809.

[6] I. W. Selesnick, "TQWT Toolbox Guide", Electrical and Computer Engineering Polytechnic Institute of New York University, (2011).

[7] I. W. Selesnick, "Sparse signal representations using the tunable Q- factor wavelet transform." SPIE Optical Engineering Applications. International Society for Optics and Photonics, (2011).

[8] R. K. Pally, "Implementation of instantaneous frequency estimation based on time-varying AR modeling”, Diss. Virginia Polytechnic Institute and State University, (2009).

[9] G. R.S. Reddy and R. Rao, "Non stationary signal prediction using TVAR model." Communications and Signal Processing (ICCSP), 2014 International Conference on. IEEE, (2014).

[10] G. Cai, X. Chen, and Z. He, "Sparsity-enabled signal decomposition using tunable Q-factor wavelet transform for fault feature extraction of gearbox", Mechanical Systems and Signal Processing, vol. 41, no. 1, (2013), pp. 34-53.

[11] S. Patidar, R. B. Pachori and N. Garg, "Automatic diagnosis of septal defects based on tunable-Q wavelet transform of cardiac sound signals", Expert Systems with Applications, vol. 42, no. 7, (2015), pp. 33153326.

[12] J. Luo, D.Yu and M. Liang, "A kurtosis-guided adaptive demodulation technique for bearing fault detection based on tunable-Q wavelet transform", Measurement Science and Technology, vol. 24, no. 5, (2013), pp. 055009.

[13] W. P. He, "sparsity-Assisted signal representation for Rotating Machinery fault diagnosis using the Tunable Q-Factor wavelet Transform with over lapping Group Shrinkage", Wavelet Analysis and Pattern Recognition (ICWP), 2014 International Conference on. IEEE, (2014).

[14] S. Chaibi, "A reliable approach to distinguish between transient with and without HFOs using TQWT and MCA." Journal of neuroscience methods, vol. 232, (2014), pp. 36-46.

[15] W. He, "Automatic fault feature extraction of mechanical anomaly on induction motor bearing using ensemble super-wavelet transform." Mechanical Systems and Signal Processing, vol. 54, (2015), pp. 457- 480 .

\section{Authors}

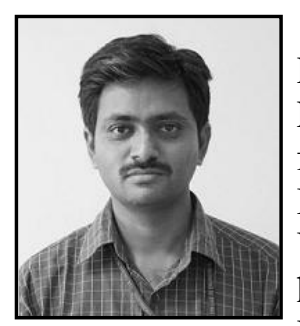

G. Ravi Shankar Reddy, is presently working as an Associate Professor in the Department of ECE, CVR College of Engineering, Hyderabad, India. He received the B.Tech Degree from Jawaharlal Nehru Technological University, Hyderabad, India, in 2001.He received the M.Tech Degree from Osmania University, Hyderabad, India, in 2004.Presently He is pursuing his Ph.D. from JNTUA, Anantapur, India, under the guidance of Dr.Rameshwar Rao. His Research area is Non-Stationary Signal Processing. 
Rameshwar Rao was Ex-Vice Chancellor of Jawaharlal Nehru Technological University, Hyderabad, India. He guided several PhD students in the area of VLSI and Signal processing. He has more than 60 publications in various National and International Journals .His research interests are in VLSI and Signal processing. 